\title{
Generation Expansion Planning Considering Integrating Large-scale Wind Generation
}

Zhang, Chunyu; Ding, Yi; Østergaard, Jacob; Wu, Qiuwei

Published in:

Proceedings of the IECON 2013

Publication date:

2013

Link back to DTU Orbit

Citation (APA):

Zhang, C., Ding, Y., Østergaard, J., \& Wu, Q. (2013). Generation Expansion Planning Considering Integrating Large-scale Wind Generation. In Proceedings of the IECON 2013 IEEE.

\section{General rights}

Copyright and moral rights for the publications made accessible in the public portal are retained by the authors and/or other copyright owners and it is a condition of accessing publications that users recognise and abide by the legal requirements associated with these rights.

- Users may download and print one copy of any publication from the public portal for the purpose of private study or research.

- You may not further distribute the material or use it for any profit-making activity or commercial gain

- You may freely distribute the URL identifying the publication in the public portal

If you believe that this document breaches copyright please contact us providing details, and we will remove access to the work immediately and investigate your claim 


\title{
Generation Expansion Planning Considering Integrating large-scale Wind generation
}

\author{
Chunyu Zhang, Yi Ding, Jacob Østergaard, Qiuwei Wu \\ Center for Electric Power and Energy \\ Technical University of Denmark \\ Copenhagen, Denmark
}

\begin{abstract}
Generation expansion planning (GEP) is the problem of finding the optimal strategy to plan the construction of new generation while satisfying technical and economical constraints. In the deregulated and competitive environment, large-scale integration of wind generation (WG) in power system has necessitated the inclusion of more innovative and sophisticated approaches in power system investment planning. A bi-level generation expansion planning approach considering large-scale wind generation was proposed in this paper. The first phase is investment decision, while the second phase is production optimization decision. A multi-objective PSO (MOPSO) algorithm was introduced to solve this optimization problem, which can accelerate the convergence and guarantee the diversity of Pareto-optimal front set as well. The feasibility and effectiveness of the proposed bi-level planning approach and the MOPSO algorithm have been verified by a numerical test system.
\end{abstract}

Keywords-Generation expansion planning; large-scale wind generation; Multi-objective PSO algorithm

\section{INTRODUCTION}

The restructure and deregulation of the global power industry have introduced fundamental changes to the practices of power system planning. Generation expansion planning (GEP) is a large-scale, nonlinear, discrete, dynamic and highly constrained optimization problem that determines which generating units should be constructed and when should be committed online over the planning horizon in such a way that installed capacity meet forecasted demand. Maximizing expected profit during the planning periods is the objective of generation companies for investing in new power plants [1].

In recent years, increasing concern for energy security, fossil fuel shortage, and the environmental challenges turn to be an urgent problem. The renewable energy sources (RES) is the key strategy to solve these issues. Wind power generation is holding the first rank in terms of use and importance. In the last decade, the growth rate of the global installed wind capacity has been about $30 \%$ per annum [2]. Denmark, Germany, and Spain are the first few countries generating 20\% of their electricity from wind turbines. However, wind resource is intermittent, stochastic and fluctuant, the large- scale integration of wind generation (WG) will bring new obstacles to power systems operation and planning.

This task supported by the EU project EcoGrid ${ }^{\mathrm{eu}}$ and Danish national project iPower.
Over the past decade, several researches and literatures have been accomplished in GEP approach considering the WG integration. The impacts of regulatory interventions on wind power expansion generation planning are investigated in [3]. The reliability of composite generation and transmission system with large-scale WG integration can be found in [4], and the effect of wind and load forecast errors on the power system expansion planning is presented in [5]. A simple approach using the annual load duration curve to evaluate the potential base and peak load savings by the installation of wind power has been presented by [6]. An integrated power generation expansion planning model towards low-carbon (green) economy is proposed by [7]. The GEP in the pool market for a multi-time period (time horizon) in regional level is solved by [8]. A multi-objective and multi-period model is proposed for integrating RES long-term expansion planning, refer to [9].

Various approaches have been proposed to deal with GEP problem, early approaches [10] for the solution include dynamic programming, merit order loading, branch-and-bound methods, and cutting-plane techniques, such as Benders decomposition. Recently, people typically conclude that the heuristic approaches can provide "high-quality" solutions in an acceptable computational time, even for large-scale problems. Several meta-heuristic methods have already been introduced in literatures [11], e.g. Genetic Algorithms (GAs), Simulated Annealing, Ant Colonies, Particle Swarm Optimal Algorithms (PSOs), Expert Systems and Fuzzy Logic and combinations of GAs and Simulated Annealing.

In this paper, based on the idea of decomposition coordination, a bi-level GEP approach considering large-scale wind generation is proposed. The first phase is investment decision, and the second phase is production optimization decision, including production simulation and maintenance scheduling. Accordingly, a MOPSO algorithm is introduced to solve this optimization problem, which can accelerate the convergence and guarantee the diversity of Pareto-optimal front set as well. Case study based on a numerical test system is conducted to demonstrate the feasibility and effectiveness of the proposed bi-level GEP approach and the MOPSO algorithm. 


\section{PROBLEM FORMULATION}

\section{A. Bi-level Problem Description}

In the GEP approach, in this paper, one generation company aims at maximizing possible profits by deciding its investments in generation capacities. These capacities determine the maximum possible amount of energy production in the market. It is easy to see that this problem has an innate two-phase structure: first investment decisions are taken and then energy productions decisions should be optimized.

The bi-level structure of this model underlines the opposing interest of the investing generation company and the market, as both interests are embodied by different objective functions. Moreover the bi-level formulation allows for the decoupling of investment and production decisions, which in reality are not taken at the same time but which are linked through the installed generation capacity, a fact that can literally be observed in the problem formulation [1].

\section{B. Phase 1: Investment decision}

The investment optimization problem is formulated in (1). The objective function represented by (1) indicates total discounted profits over the planning period [3].

$$
\begin{aligned}
& \operatorname{Max} O_{P 1}=\operatorname{Max} E\left[\sum_{k=0}^{T}\left[(1+r)^{-k} B_{k}\left(G_{k}, G_{w k}, D_{k}, G_{e k}, G_{w e k}, M_{k}\right)\right]\right] \\
& =\operatorname{Max} E\left[\sum_{k=0}^{T}\left[(1+r)^{-k}\left[E\left(B_{e k}\right)+E\left(B_{f k}+B_{r k}\right)-C_{i k}-C_{\imath k}-C_{t k}\right]\right]\right] \\
& \text { s.t. } \quad \sum_{x=1}^{X} P_{G e, x}+\sum_{y=1}^{Y} P_{G w e, y} \leq D_{k} \\
& \quad P_{G e, \min } \leq P_{G e} \leq P_{G e, \max } \\
& \quad P_{G w e, \min } \leq P_{G w e} \leq P_{G w e, \max } \\
& P_{G}=\sum_{x=1}^{X} P_{G e, x}+\sum_{y=1}^{Y} P_{G w e, y} \\
& M_{k} \leq M_{c}
\end{aligned}
$$

where $E$ is the expected benefits of investment planning; $r$ is the discounted rate; $k$ is the time step (yearly); $B_{k}$ is the expected net profit. In each scenario $k, B_{e k}$ is the investor's revenues obtained in the power market; $B_{f k}$ and $B_{r k}$ are the revenue of wind firms obtained through Feed-in-tariff incentive and no carbon generation reward, respectively; $C_{i k}$ is the investment cost; $C_{v k}$ is the operation cost; $C_{c k}$ is the $\mathrm{CO}_{2}$ taxes; $G_{k}$ is the power generation except wind power; $G_{w k}$ is the WG; $D_{k}$ is the demand; $G_{w e k}$ is the WG expansion; $G_{e k}$ is the other generation expansion; $Y$ is the number of candidate $\mathrm{WG}$ companies; $X$ is the number of other candidate generation companies; $P_{G w e}$ is the power of $\mathrm{WG} ; P_{G e}$ is the power of other generation; $P_{G}$ is the total power generation; $M_{k}$ is the price of electricity; $M_{c}$ is the price cap.

The formulation and calculation of $B_{e k}, B_{f k}, B_{r k}, C_{i k}, C_{v k}$, and $C_{c k}$ could be found in [3]. (2) is the demand constraint. (3) and (4) are the bounds upon the decision variables. (5) is an auxiliary constraint that represents total power generation of every company for each scenario and load level. (6) is the price cap constraint to prevent increasing the price of electricity.

\section{Phase 2: Production decision}

For each scenario $k$, the production optimization decision could be divided into two sub-problems, i.e. production simulation and maintenance scheduling.

\section{1) Production simulation}

The objective of production simulation is the minimum expectation of generation curtailment with voltage and thermal constraints, which can be depicted as follows:

$$
\begin{array}{ll}
\text { Min } & O_{P 21}=\sum P_{G C i} \\
\text { s.t. } & P_{G i}-P_{L i}-P_{G C i}=P_{I N i}(U, \theta, F) \\
& Q_{G i}-Q_{C i}-Q_{L i}-Q_{G C i}=Q_{I N i}(U, \theta, F) \\
& S_{i j} \leq S_{i j, \max } \\
& U_{i, \min } \leq U_{i} \leq U_{i, \max } \\
& P_{G i, \min } \leq P_{G i} \leq P_{G i, \max } \\
& Q_{C i, \min } \leq Q_{C i} \leq Q_{C i, \max } \\
& Q_{G C i}=f\left(P_{G C i}\right) \\
& F_{L, \min } \leq F_{L} \leq F_{L, \max }
\end{array}
$$

where, at the $i$ th node, $P_{G i}$ and $Q_{G i}$ are the active and reactive generation; $P_{L i}$ and $Q_{L i}$ are the active and reactive load; $P_{G C i}$ and $Q_{G C i}$ are active and reactive generation curtailment or increase; $Q_{C i}$ is the reactive power generated/absorbed by a reactive compensation equipment; $P_{I N i}$ and $Q_{I N i}$ are the injection active and reactive power; $S_{i j}$ is the load flows of branch $i j ; \theta$ is the node voltage angle; and $U$ is the node voltage magnitude; $F_{L}$ is the tap setting of the tap changer $L$.

The optimization object (7) need subject to not only power flow equation constraints ((8) and (9)), but also the branch thermal constraint (10) and network voltage limits (11). The amount of active generation curtailed will be limited by the capacity of generation connected (12). Reactive power support is limited by the capacity of reactive compensation equipments installed (13). Reactive power curtailment may be correlated with the active power curtailment, which is modeled through (14). The tap changer setting will be optimized and can vary within the bounds given by (15).

\section{2) Maintenance scheduling}

The objective of maintenance scheduling (16) is the minimum risk of power system during generation maintenance (17) with the duration (18), frequency (19) and numbers (20) constraints, which can be formulated as follows:

$$
\operatorname{Min} O_{P 22}=N^{-1} \sum_{n=1}^{N}\left(P_{R, n}-N^{-1} \sum_{n=1}^{N}\left(P_{R, n}\right)\right)^{2}
$$




$$
\begin{array}{ll}
\text { s.t. } & P_{R, n} \geq P_{R, \min } \\
& I_{n} \leq t_{G, n} \leq V_{n} \\
& t_{G, n+1}-\left(t_{G, n}+t_{D, n}\right) \geq \lambda \\
& N_{t_{G}, n} \leq N_{t_{G}, n, \max }
\end{array}
$$

where $N$ is the number of generations; In each maintenance duration $t_{D, n}, P_{R, n}$ is the LOLP (risk) of power system; $P_{R \text {, min }}$ is the minimum allowable risk of power system; $t_{G, n}$ is the start time of maintenance; $I_{n}$ and $V_{n}$ are the earliest and latest start time; $\lambda$ is the minimum interval of twice maintenance; $N_{t G, n}$ is the number of simultaneous maintenance.

\section{MethodOLOGY}

\section{A. Classical PSO}

Particle swarm optimization is a relatively new technique for optimization of continuous non-linear functions. The individuals in a PSO have their own positions and velocities. Each particle moves in the search space with velocity, which is dynamically adjusted and balanced based on its own best movement $\left(p_{b}\right)$ and the best movement of the group $\left(g_{b}\right)$. If the best previous position of the $i$-th particle is recorded and represented as $p_{i}=\left(p_{b 1}, p_{b 2}, p_{b 3}, \ldots, p_{b d}\right)$ and the global best position is recorded and represented as $g_{i}=\left(g_{b 1}, g_{b 2}, g_{b 3}, \ldots\right.$, $\left.g_{b d}\right)$, the modification of velocity $\left(v_{i d}\right)$ and position $\left(x_{i d}\right)$ of the $i$ th particle can be calculated by the current velocity and the distance from $p_{i}$ to $g_{i}$ as shown in the following formulas,

$$
\begin{gathered}
v_{i d}=w \cdot v_{i}+c_{1} \cdot r_{1}\left(p_{i}-x_{i d}\right)+c_{2} \cdot r_{2}\left(g_{i}-x_{i d}\right) \\
x_{i d}=x_{i d}+v_{i}
\end{gathered}
$$

where $w$ is known as the inertia weight, $c_{1}$ and $c_{2}$ are two positive constants, $r_{1}$ and $r_{2}$ are random numbers in the range of $[0,1]$.

The important part in MOPSO is to determine the global best particle $g_{i}$ for each particle $i$ of the population. In single objective PSO, the global best particle is determined easily by selecting the particle in the best position. In multi-objective optimization problems, each particle of the population should select its global best particle from the set of Pareto-optimal solution.

\section{B. Two-phase MOPSO}

Different from normal multi-objective optimization methods that either pay attention to the convergence rate with the cost of worse diversity of solutions or pay attention to the diversity of solutions with the cost of lower convergence rate and more computing time, the tow-phase guide MOPSO method introduced in this paper takes both the convergence rate and the diversity of solutions into account. The Sigma method is adopted to the first half of the evolution because of its fast convergence rate, and an approximated front of Pareto is found. And then an introduced ideal optimal particle method is adopted to the remaining half of the evolution that will promote the diversity of solution. This method can both obtain higher convergence rate and ensure better diversity of solutions.

\section{1) The steps of two-phase MOPSO}

Step 1: Initialize the parameters of two-phase MOPSO, including size of the swarm $P$, capacity of the archive $A$, PSO coefficients $w, c_{1}$ and $c_{2}$, and maximum iterations $Z, t=0$;

Step 2: Randomly initialize the position and velocity of each particle in set $P$ and set $A$. Set the initial position as the individual best position $p_{i}$ of each particle;

Step 3: For $t=1$ to $Z$,

- Update archive,

- Select the global bests $\left(g_{i}\right)$ from $A$ for each particle in the set $P$ based on the strategy of two-phase guided,

- Update position and velocity of every particle according to the $(21)-(22)$,

- If $t<0.85 Z$, the particle is mutated according to the strategy,

- $\quad$ Each particle in set $P$ has a new location, if the current location is dominated by its personal best location $\left(p_{i}\right)$, then the previous location is kept, otherwise, the current location is set as the personal best location. If the particles are mutually non-dominated, one particle is selected randomly;

- $\quad$ End.

\section{2) Strategy of archiving}

In normal MOPSO optimization algorithm, the global solution will be stored in the external archive. If the size of the archive exceeds the maximum size limit, it will be truncated using the diversity consideration by crowding distance mechanism to maintain the diversity of non-dominated solutions.

The crowding distance of a solution provides the density of solutions around this solution. The crowding distance of a solution is the average distance between its two neighboring solutions. The boundary solutions which have the lowest and the highest objective function values are given an infinite crowding distance values so that they are always selected. The final crowding distance value of a solution is computed by adding the entire individual crowding distances in each objective function.

After all population members in the Pareto-optimal set are assigned a distance metric, each solution can be compared with other solutions in the set. A solution with a smaller crowding distance indicates that it is more crowded than other solutions and the diversity of the solution is smaller. On the contrary, a solution with larger distance indicates that the diversity of solutions is larger.

Therefore, a solution with larger crowding distance will be reserved and one with smaller crowding distance will be deleted from the external archive to make solutions diversified.

3) Strategy of two-phase guided 
The selection of $g_{i}$ is the key of PSO algorithm. For multiobjective problems, $g_{i}$ is related to the convergence speed of the algorithm and the diversity of solution. A two-phase guide $g_{i}$ selection strategy is introduced in this paper. Sigma method is adopted to the first half of the evolution because of its fast convergence rate, and an approximated front of Pareto is found. And then, an introduced ideal optimal particle method is adopted to the remaining half of the evolution, which will promote the diversity of solution.

\section{a) Sigma method}

The Sigma method involves choosing the guide particle based on the similarity of the angular position in objective space. This method was proposed by Mostaghim and Teich [12], which was proved to have good convergence speed. For two-dimensional objective space, $\delta$ is defined as

$$
\delta=\frac{f_{1}^{2}-f_{2}^{2}}{f_{1}^{2}+f_{2}^{2}}
$$

The guide particle of particles in set $P$ is the particle that has the closest $\delta$ in set $A$.

\section{b) Ideal optimal particle method}

Defined $f_{j}^{\prime}$ is regarded as the optimal value of the $j$ th objective, and the point $\left(f_{1}, f_{2}, \ldots, f_{n}\right)$ is called ideal point. The optimal particle $x_{I}$ of the following equation is the ideal optimal particle

$$
\min \varphi\left(f\left(x_{I}\right)\right)=\sqrt{\sum_{j=1}^{n}\left(f_{j}\left(x_{I}\right)-f_{j}^{\prime}\right)^{2}}
$$

For a complex multi-objective optimization problem, the optimal value of the each objective and the corresponding optimal point tends to be unknown advance. In this paper, the previous optimal value of each objective in iterative process is regarded as $f_{j}$.

The process in which the guide particles are selected from set $A$ is described as the following steps:

- Calculate the optimal value $f_{j}^{\prime}$ and the corresponding optimal particle $x$ ' in set $A$;

- According to (24), obtain the ideal optimal particle $x_{I}$;

- Compute the average of the optimal particle $x^{\prime}$ and the ideal optimal particle $x_{I}$, and regard and average value as $g_{i}$.

The value $g_{i}$ represents the optimal information of the each objective, achieves the global optimization of the algorithm, and is helpful to achieve the local depth search.

\section{c) Selecting guided particle strategy}

Sigma method which has faster convergence rate is adopted to the first $n_{1}$-th generation of the evolution, and the ideal optimal particle method is adopted to the remaining $\left(Z-n_{1}\right)$-th generation.

\section{4) Strategy of mutation}

The use of mutation operator in this MOPSO is needed because the algorithm may converge to local optimal fronts.
Mutation probability $\left(P_{m}\right)$ is reduced with the iteration of the algorithm according to

$$
p_{m}=1-\frac{C_{g}}{Z}
$$

where $C_{g}$ is the number of current generation. For each particle, the variable $m_{r}$ is a random number in the range of $[0,1]$.

If $m_{r}<P_{g}$, the particle is randomly selected for mutation according to

$$
x_{i}=\delta \cdot \mu \cdot\left(1-m_{r}\right) \cdot v_{i}+x_{i-1}
$$

where $\mu$ points out the direction in mutation and $\delta$ controls the distance covered by a jump. In this paper, $\mu=3$ and $\delta$ is set as \pm 1 randomly. If the solution is beyond its boundary by mutation, it is moved to the corresponding boundary.

\section{GEP program flow}

The major GEP modules and the general flow of the program are shown in Fig. 1. In the planning procedure, several possible planning schemes could be generated. Then the best one is selected among them considering different factors. The factors concerned are so many that sometimes it is difficult for a planner to have all things considered merely by his past experiences.

Therefore, a Fuzzy satisfying decision making approach was introduced in this program, including three steps: boundary decision, normalization and decision analysis, which have been depicted in detail in [13].

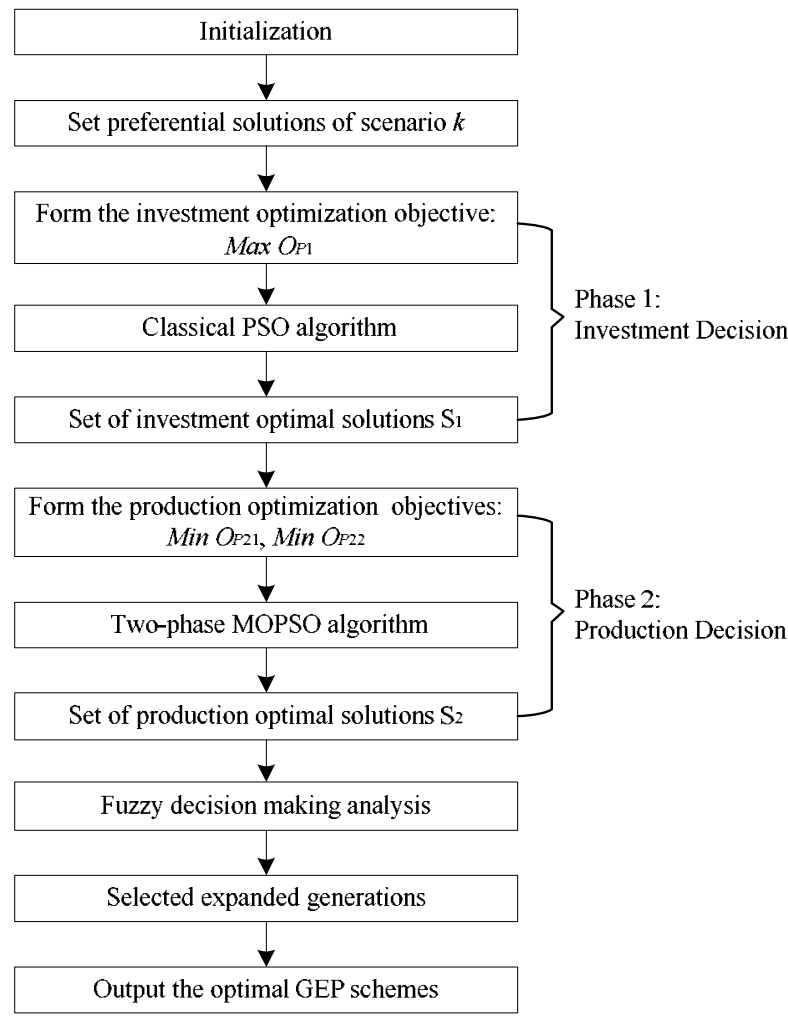

Fig. 1. The bi-level GEP program flow 


\section{CASE StUdy}

The proposed framework has been implemented on a test system. Initial data of the test system are collected from [14] and [15]. The test system has 17,000 MW generations as total installed capacity. The elastic demand is considered in this study. The planning horizon is assumed to be 5 years, and the probabilistic distribution function of wind power generation is evaluated in each year as shown in [3].The electricity market is assumed to be consisted by four companies, generation technologies regarding the ownership of the units by different companies are shown in TABLE I.

TABLE I. GENERATION TECHNOLOGIES

\begin{tabular}{|c|c|c|c|c|c|}
\hline Company & $\mathbf{C}_{\mathbf{1}}$ & $\mathbf{C}_{\mathbf{2}}$ & $\mathbf{C}_{\mathbf{3}}$ & $\mathbf{C}_{\mathbf{4}}$ & $\mathbf{C}_{\mathbf{5}}$ \\
\hline $\begin{array}{c}\text { Generation } \\
\text { technology }\end{array}$ & Nuclear & $\begin{array}{c}\text { Oil/ } \\
\text { Steam }\end{array}$ & $\begin{array}{c}\mathrm{Coal} / \\
\text { Steam }\end{array}$ & CCGT & Wind \\
\hline $\begin{array}{c}\text { Variable cost } \\
\text { \$/MWh) }\end{array}$ & 1.83 & 18.89 & 7.07 & 10.95 & 0 \\
\hline $\begin{array}{c}\text { Capacity } \\
\text { (MW) }\end{array}$ & 4,000 & 2,200 & 7,000 & 3,000 & 800 \\
\hline $\begin{array}{c}\text { GEP candidate } \\
\text { capacity }(\boldsymbol{M W})\end{array}$ & 400 & 200 & 300 & 50 & 50 \\
\hline $\begin{array}{c}\text { Lifetime } \\
\text { (year) }\end{array}$ & 40 & 40 & 40 & 20 & 30 \\
\hline $\begin{array}{c}\text { CO } \boldsymbol{2} \\
(\text { lbs } / \boldsymbol{M W})\end{array}$ & 0 & 1,638 & 1,840 & 889 & 0 \\
\hline
\end{tabular}

The discount rate is set to be $6 \%$, annual growth of the demand is supposed to be $6 \%$, the price cap is considered $80 \$ / \mathrm{MWh}$, and load coefficients are assumed to be 2, 1.5 and 1 for peak, medium and base-load sub-period. Furthermore, the impacts of Feed-in-tariff incentives and no carbon generation reward on GEP schemes are also considered in this case study, which is assumed to be $30 \$ / \mathrm{MWh}$ and $0.01 \$ / \mathrm{lbCO}_{2}$, respectively.

TABLE II. GEP SCHEMES OF THE CASE

\begin{tabular}{|c|c|c|c|c|c|c|}
\hline \multicolumn{2}{|c|}{ Year } & $\mathbf{Y}_{\mathbf{1}}$ & $\mathbf{Y}_{\mathbf{2}}$ & $\mathbf{Y}_{\mathbf{3}}$ & $\mathbf{Y}_{\mathbf{4}}$ & $\mathbf{Y}_{\mathbf{5}}$ \\
\hline \multirow{4}{*}{ Schemes } & $\mathbf{C}_{\mathbf{1}}$ & - & $1 \times 400$ & $1 \times 400$ & $1 \times 400$ & $1 \times 400$ \\
\cline { 2 - 7 } & $\mathbf{C}_{2}$ & - & - & - & - & - \\
\cline { 2 - 7 } & $\mathbf{C}_{\mathbf{3}}$ & - & - & - & - & - \\
\cline { 2 - 7 } & $\mathbf{C}_{4}$ & - & - & - & - & - \\
\cline { 2 - 7 } & $\mathbf{C}_{5}$ & $6 \times 50$ & $13 \times 50$ & $16 \times 50$ & $21 \times 50$ & $24 \times 50$ \\
\hline $\begin{array}{c}\text { Wind } \\
\text { penetration (\%) }\end{array}$ & 6.36 & 9.54 & 12.78 & 17.14 & 21.24 \\
\hline $\begin{array}{c}\text { Wind revenues } \\
(\boldsymbol{M} \$ \text { ) }\end{array}$ & 1124.58 & 1768.32 & 2958.48 & 3785.97 & 5201.23 \\
\hline $\begin{array}{c}\text { Average annual } \\
\text { price (\$/MWh) }\end{array}$ & 63.68 & 67.03 & 68.09 & 68.33 & 68.19 \\
\hline
\end{tabular}

The GEP schemes of this case study are presented in TABLE II, also including the wind penetration index, wind revenues, and average annual price. By investigating the results of Table 2, it is observed that the wind penetration has a notable increase from $6.36 \%$ to $21.24 \%$ (in the $5^{\text {th }}$ year), the WG growth rate is around $4 \%$ per year. Obviously, the rapid growth of wind revenues will definitely promote more WG companies to invest. The average annual price increased every year is due to the WG company premium has been accumulated.

\section{CONCLUSION}

A bi-level GEP approach considering large-scale wind generation is proposed, the first phase is investment decision, and the second phase is production optimization decision, including production simulation and maintenance scheduling. And its solving algorithm based on the two-phase MOPSO is also induceded in this paper. The planning results of the numerical test system show that, for a large and practical system, the proposed bi-level GEP method can effectively enhance the WG installation in any arbitrary year before the horizon. Considering economic and reliability, the best planning schemes can be put forward by the introduced twophase MOPSO, which shows its superiority as well.

\section{ACKNOWLEDGMENT}

The authors gratefully acknowledge the financial supports and the strategic platform for innovation \& research provided by EU project EcoGrid ${ }^{\mathrm{eu}}$ and Danish national project iPower.

\section{REFERENCES}

[1] S. Wogrin, E. Centeno, and J. Barquin, "Generation capacity expansion in liberalized electricity markets: a stochastic MPEC approach," IEEE Trans. Power Systems, vol. 26, pp. 2526-2532, Nov. 2011.

[2] Y. Ding, P. Wang, P. C. Loh, and Q. Wu "Long-term reserve expansion of power systems with high wind power penetration using universal generating unction methods," IEEE Trans. Power Systems, vol. 26, pp. 766-774, Nov. 2011.

[3] E. Alishahi, M. P. Moghaddam, and M. K. Sheikh, "An investigation on the impacts of regulatory interventions on wind power expansion in generation planning," International Journal of Energy Policy, vol. 39, pp. 4614-4623, May. 2011.

[4] H. Saboori, M. Mohammadi, and R.Taghe, "Composite generation and transmission expansion planning considering the impact of wind power penetration," in Proc. 2011 IEEE APPEEC, pp. 574-580, Jun. 2011.

[5] H. Yu, C. Y. Chung, K. P. Wong, and J. H. Zhang, "A chance constrained transmission network expansion planning method with consideration of load and wind farm uncertainties," IEEE Trans. Power Systems, vol. 24 pp. 1568-1576, Aug. 2009.

[6] G. Mel. and B. Rangan, "Analysis of impacts of wind integration in the Tamil Nadu grid," International Journal of Energy Policy, vol. 39, pp. 3693-3700, May. 2009.

[7] F. Careri, C. Genesi, P. Marannino, M. Montagna, M. Rossi and I. Siviero, "Generation Expansion Planning in the age of green economy," IEEE Trans. Power Systems, vol. 26, pp. 2214-2223, Nov. 2011.

[8] S. M. Moghddas-Tafreshi, H. A. Shayanfar, A. S. Lahiji, A. Rabiee and J. Aghaei, "Generation expansion planning in Pool market: A hybrid modified game theory and particle swarm optimization," International Journal of Energy Conversion and Management, vol. 52, pp. 1512-1519, Nov. 2010.

[9] A M. R. Sierra, and C. A. Coello, "Multistage expansion planning of generation and interconnections with sustainable energy development criteria: A multiobjective model," International Journal of Electrical Power and Energy System, vol. 33, pp. 258-270, Aug. 2011.

[10] H. Bai and B. Zhao, "A survey of the application of swarm intelligence computation to electric power systems," Proc. 6th World Congr. Intelligent Control and Automation, pp. 7587-7591, 2006.

[11] A. J. C. Pereira, and J. T. Saraiva, "Generation expansion planning (GEP): A long-term approach using system dynamics and genetic algorithms (GAs)," International Journal of Energy, vol. 36, pp. 51805199, Jul. 2011.

[12] S. Mostaghim, J. Teich, "Strategies for finding good local guides in multi-objective particle swarm optimization (MOPSO)," in Proc. 2003 IEEE Swarm Intelligence Symposium, pp. 26-33. 
[13] T. S. Chung, K. K. Lee, G. J. Chen, J. D. Xie, and G. Q. Tang, "Multiobjective transmission network planning by a hybrid GA approach with fuzzy decision analysis," International Journal of Electrical Power \& Energy Systems, vol. 25, pp. 187-192, Jun. 2003

[14] S. Kannan, S. Baskar, J. D. McCalley, and P. Murugan, "Application of NSGA-II Algorithm to generation expansion planning," IEEE Trans. Power Systems, vol. 24 pp. 454-461, Aug. 2009.
[15] H. Tekiner, D. W. Coit, and F. A. Felder, "Multi-period multi-objective electricity generation expansion planning problem with Monte-Carlo simulation," International Journal of Electric Power System Research, vol. 80, pp. 1394-1405, Jul. 2010. 